\title{
HYPOTHESIS
}

\section{Idiopathic osteoporosis: an evolutionary dys-adaptation?}

\author{
C Alexander
}

\begin{abstract}
Osteoporosis is characterised by simultaneous net bone growth and net resorption on different surfaces, suggesting that systemic factors are not the sole explanation for the findings. The main clinical consequence is fracturing in the largely trabecular bones of the spine, hip, and radius, and the key problem in these areas is finding an explanation for the preferential loss of transverse trabeculae. In normal bone, local maintenance depends on a negative feedback response to intermittent compression strain, and it is concluded, from biomechanical analysis of the response required to achieve negative feedback, that a preferential loss of transverse trabeculae is indicative of a selective deficiency of radial compression loading. The only significant source of radial compression in humans is the induced strain produced by axial tension. This is a necessary component of the lifestyles of quadrupeds and arboreal primates, but in humans occurs only on the convex side when the bone is offset loaded. The resulting strain is a function of the range of movement. It is suggested that the asymmetrical pattern of bone loss in cortical and trabecular osteoporosis reflects chronic underuse of movement range, resulting from the adoption of a bipedal lifestyle. Exercise regimens based on using the whole of the available movement range should better prepare the skeleton to adjust to other factors hostile to bone maintenance.

(Ann Rheum Dis 2001;60:554-558)
\end{abstract}

Department of

Anatomy with

Radiology, School of

Medicine, University

of Auckland, New

Zealand

C Alexander

Correspondence to:

Professor C Alexander,

School of Medicine,

University of Auckland,

Private Bag 92019,

Auckland, New Zealand

c.alex@xtra.co.nz

Accepted 13 December 2000 the disease is regarded as multifactorial. It affects both sexes, and in women the bone loss is sharply accelerated at the menopause, but there is evidence that it starts before that time, perhaps as soon as growth has ceased. ${ }^{6-10}$ The main clinical consequence is fracturing in the largely trabecular bones of the spine, hip, and radius, and the dominant clinical setting is post-menopausal hormone deficit.

Although there is no dispute about the severe catabolic effect of this hormone change, there are two pieces of evidence which suggest that systemic factors cannot, by themselves, explain all the findings. The first was pointed out by Villanueva et al. ${ }^{11}$ When the disease affects long bones, resorption of the inner cortex is accompanied by simultaneous and continuing bone accretion on the outer surface, though both are exposed to the same hormonal and chemical environment. Similarly, in trabecular osteoporosis, early resorption of transverse trabeculae is initially accompanied by retention and in many cases actual thickening of axial trabeculae. $^{712-15}$ This is seen in all bones but is particularly evident in vertebrae. The pattern of bone loss is inhomogeneous as well as anisotropic, and the selective thickening may not be seen in all sections or in small regions of interest (figs 1 and 2), ${ }^{1316}$ but it is commonly present when whole vertebrae are scanned, clinically or experimentally, and its reality is not in dispute. It has the same implications as the asymmetrical loss in long bones. Blood levels of calcium and hormone are the same for all surfaces, and if there is sufficient for some vertical trabeculae to thicken, the antecedent and concurrent loss of transverse trabeculae cannot be ascribed solely to their deficiency.

The eventual but asynchronous loss of axial trabeculae, and the healed fractures commonly found in the survivors ${ }^{17}$ are predictable consequences of Euler's Law. The strength of a loaded strut varies inversely with the square of its unsupported length, ${ }^{3}$ and premature loss of transverse trabeculae will compromise both unsupported vertical trabeculae and the cortices at the end of long bones such as the femoral neck. There is, however, no simple explanation for the preferential loss of the originally thicker transverse trabeculae, and as Twomey et al point out, ${ }^{18}$ it is this selective resorption which constitutes the crux of the problem. Any acceptable hypothesis has to explain it.

These asymmetries constitute a local failure of bone maintenance, specifically the failure to 


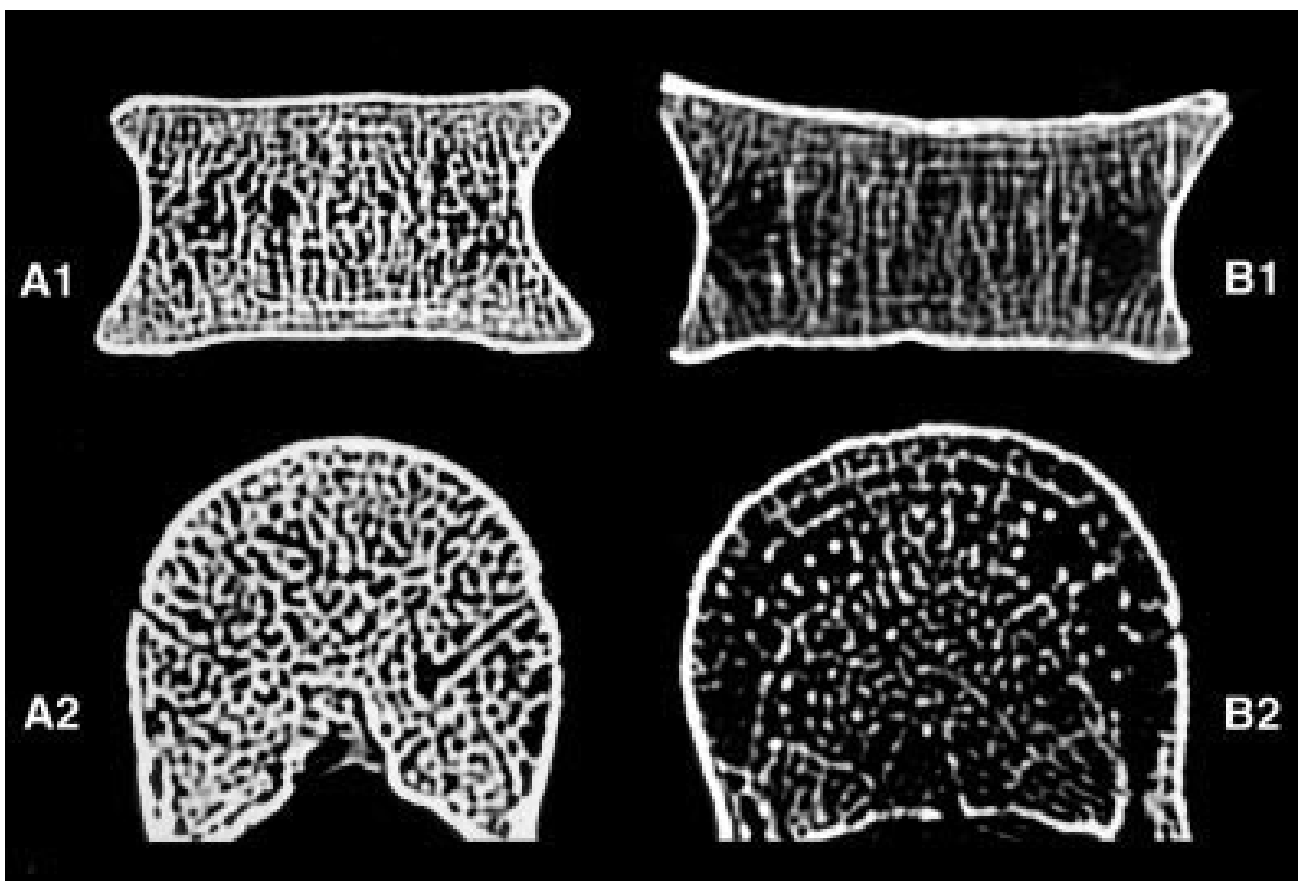

Figure 1 Coronal and transverse computed tomographic (CT) scans of dry vertebrae, $1 \mathrm{~mm}$ slice thickness, $120 \mathrm{kV}, 250$ $m A$. Left, a young adult vertebra, mean (SD) $x$ ray absorption -570 (102) Houndsfield Units (HU). Right, an osteoporotic vertebra, $x$ ray absorption -827 (103) HU. The right hand figures are representative middle third scans from a sample of 61 triplanar CT scans made across three osteoporotic vertebrae. The transverse scan B2 shows substantial loss of radial and annular trabeculae. There is some loss of axial trabeculae with thickening of several unsupported survivors. The pattern of bone loss is not uniform across the transverse section, and differs from that seen in coronal and sagittal sections.

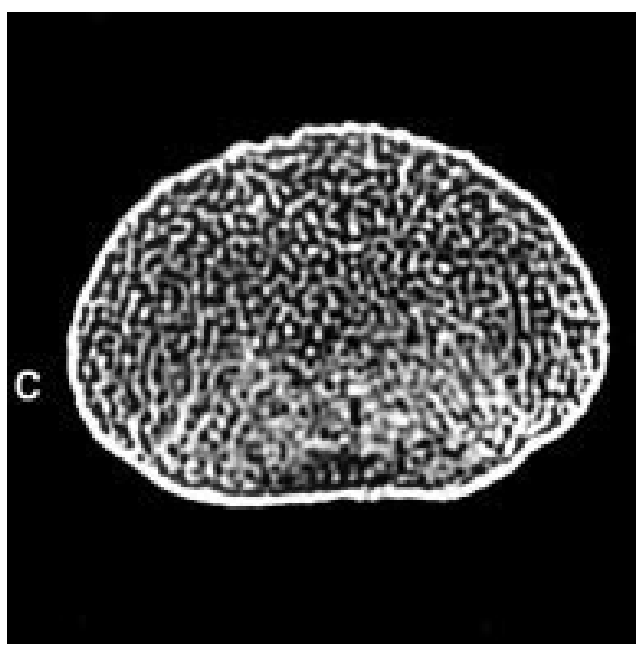

Figure 2 A transverse $1 \mathrm{~mm}$ computed tomographic section of the same vertebra as in fig 1, B2, taken $8 \mathrm{~mm}$ more distally. It shows little sign of osteoporosis with no detectable axial thickening. Trabeculae near the tension-resisting end plate are better preserved and show less anisotropy.

maintain the thickness of cortices and of transverse trabeculae, and they require a local explanation. There is increasing evidence that the dominant factor in local bone maintenance is dynamic loading, ${ }^{19-21}$ but not all loads are effective in this role. Experiments on static compression report a net bone loss. ${ }^{19} 22$ Intermittent tension can stimulate longitudinal osteogenesis-for example, in the healing phase after osteotomy, but it is vectored to occur in the line of tension, ${ }^{23-25}$ and there are no reports of tension causing transverse thickening in intact bones. Bones exposed to persisting tension, as on the convex side of the bent rat's tail or in human scoliosis, become atrophic. ${ }^{26}{ }^{27}$ Loading in torsion is mildly anabolic, ${ }^{20}$ but intermittent axial compression is a strong stimulant, producing new bone on both the compression and tension cortices of the loaded bone. ${ }^{19} 22$ 28-31 Human subjects exposed to intermittent axial loading exercises also show an anabolic response. ${ }^{32}{ }^{33}$ It appears then that the most likely cause of a local failure of bone maintenance in normal subjects is a local deficiency of intermittent compression loading.

There are, however, two requirements before intermittent compression can be effective. Firstly, the rate of strain $^{29} 31$ and its peak magnitude $^{30}{ }^{34}$ must exceed critical thresholds before osteogenesis is stimulated. It appears that if these requirements are met, the total amount of loading needed is relatively slight; Rubin and Lanyon found that as few as four movements a day were sufficient to prevent osteoporosis. ${ }^{35}$ Secondly, to meet the requirements of negative feedback, the response must operate to reduce the error signal, ${ }^{36}$ which means that the resulting bone formation must be vectored. If, for example, a long bone exposed to excess axial load responded by increasing in length, the change would increase the strain, resulting in positive feedback. Only an increase in thickness will reduce the strain and hence the error signal. Both experiment and clinical experience confirm this vectoring; the concave side of the bowed tibia or rat's tail segment increases in cortical thickness, not in length. ${ }^{26}$ To reduce the signal, the plane of the response must be at right angles to the vector of the load. 
Strut under compression

A

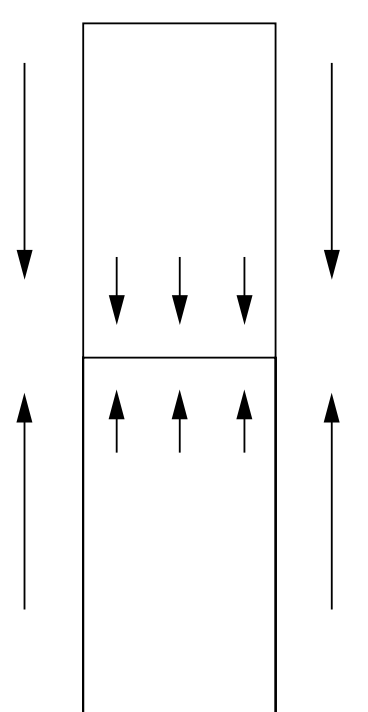

B

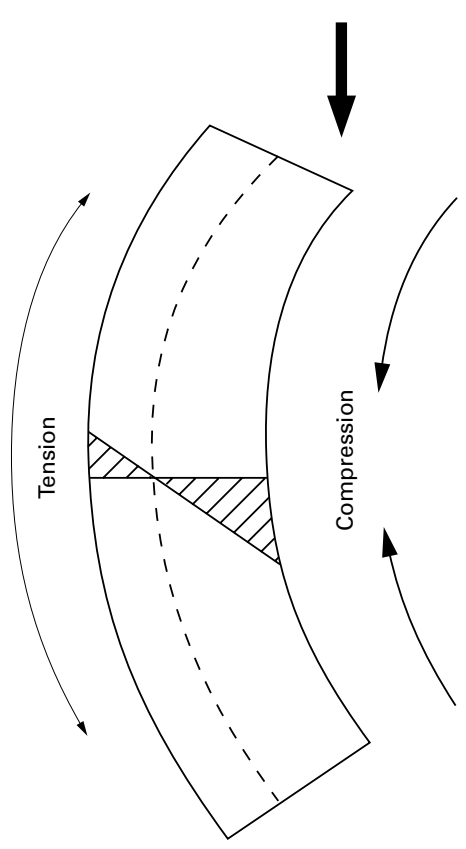

Figure 3 Strain distribution in a bent strut. Both compression and tension strains are maximal at the cortical surface and a function of the degree of bending. The longitudinal tension strain induces a transverse compression strain on the convex side of the neutral zero-strain axis.

Axial compression

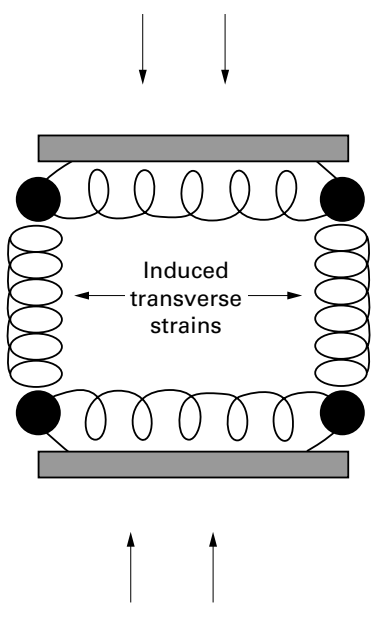

Axial tension
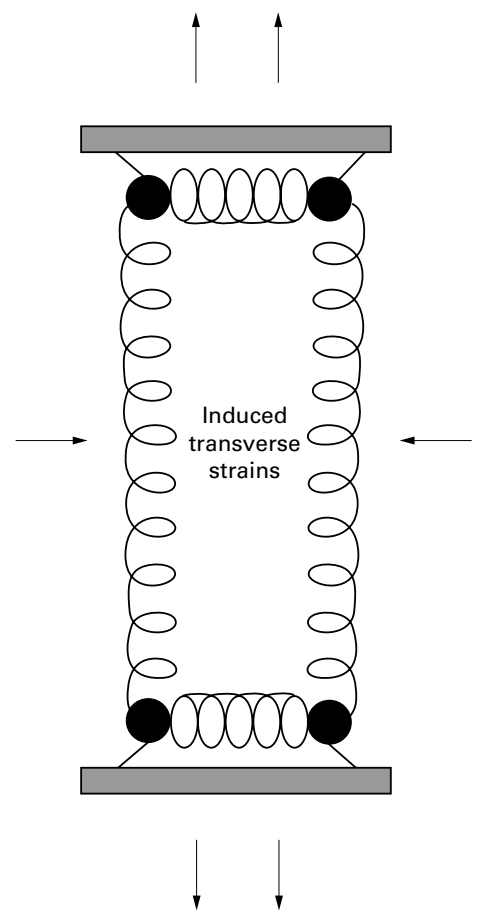

Figure 4 Transverse strains induced by longitudinal strains on an elastic body.
It follows that the plane of bone loss identifies the vector of the missing strain. The loss of transverse cortex indicates that the axial compression strain has been deficient in rate, magnitude, or both. Similarly, the selective loss of thickness of transverse trabeculae indicates a strain deficiency at right angles to the plane of loss - that is, a radial compressive strain in the transverse plane. In the first case, as long bones are normally subjected to alternating compression and tension strains on opposing surfaces, ${ }^{37}$ the rate and magnitude of the strains can be increased by impact loading, ${ }^{32}$ or, with less risk, by increasing the range of movement. Even a tiny offset causes a disproportionately large increase in strain, proportional to the offset and hence to the range of movement (fig 3). ${ }^{28}{ }^{38}$ For a typical loading situation as it might apply in the human spine, it can be shown that increasing the loading offset by as little as $5 \mathrm{~cm}$ and two degrees increases the axial compression strain by more than an order of magnitude. A further increase to 30 degrees increases the magnitude by a further factor of three, and the strain rate by a factor of 15 for the same frequency. These increases would be augmented by any coincident muscle contraction. If cortices atrophy, it seems likely that the missing factor is use of movement range.

Identifying the missing activity in trabecular osteoporosis is less straightforward; there are no muscle groups applying a concentric radial compression force to vertebrae or the ends of long bones. Apart from the exceptional circumstance of the Valsalva manoeuvre, direct loading in this direction must be unusual. There is, however, a less obvious source of radial compression.

\section{Induced strains}

When a strut is exposed to axial loading in compression or tension, secondary "Poisson" strains, expansile or contractile, are induced within the strut at right angles to the external load (fig 4). The compressed trellis and stretched sock are everyday examples. The axially loaded spine or long bone is a strut under compression with a tension resisting sleeve, and the known increase in vertebral and long bone diameter with age may reflect the operation of this induced expansile strain. In bone, which has a Poisson ratio of the order of 0.4, these induced strains are not trivial, and as the dominant load is usually close to axial, they are maximal in, or close to, the transverse plane. $^{3739}$ It follows then that axial tension would provide the radial compression needed for maintenance of the thickness of transverse trabeculae. Pure axial tension is not an option for the non-arboreal primate, but when a strut is bent or offset loaded, as is normally the case, the axial tension on the convex side of the neutral axis shows the same disproportionate increase as was noted above for axial compression, and the induced transverse compression increases in like manner. The induced strains in cancellous bone are about 20 times as great as in compact bone, owing to its lower modulus of elasticity, and again are a function of the range of movement. ${ }^{28} 39$

It appears then that under normal loading conditions, the compression on the concave side is providing the strain needed to maintain the ipsilateral cortex and axial trabeculae, while the tension on the opposite side is maintaining the contralateral transverse trabeculae. As the main deficiency in clinical osteoporosis is loss 
of transverse trabeculae, it is the axial tension which is the more important, and in practical terms this can only be increased by increasing the movement range.

Is there any evidence that humans are in fact underusing the design envelope for joint movement? The acrobatic foraging of the arboreal ape, ranging through bipedal and quadrupedal walking, running, climbing, and brachiation, ${ }^{40} 41$ requires far more use of its spinal movement range than is needed by the biped, ${ }^{42}$ and the pendulum-like action of brachiation makes exceptional demands on shoulder, elbow, and fingers. ${ }^{43} 44$ In the quadruped, the propulsive force on locomotion is axial, and produces alternating tensile and compressive strains on opposing vertebral margins. ${ }^{45}$ In animals with flexible spines this is accompanied by visible oscillation in coronal or sagittal planes, depending on gait. ${ }^{46}$ The human, with reported spinal movement ranges of 154 degrees in flexion-extension, 107 degrees in lateral flexion, and 87 degrees in rotation, ${ }^{47}$ is unequivocally in the flexible-spine subset, but with the propulsive force now at right angles to the $y$ axis, the oscillating strains intrinsic to the quadrupedal gait are no longer necessary for progression. Apart from a few degrees of rotation, spinal flexions are not recorded in studies of human walking. ${ }^{48}$ There is, in addition, increasing evidence that humans underuse the arc available in some peripheral joints. ${ }^{49}$

These data suggest the parsimonious hypothesis that the initiating factor in both cortical and trabecular osteoporosis is habitual underuse of movement range, resulting from abandonment of arboreal and quadrupedal lifestyles. This follows the concept of Lanyon that loading is the effector for bone homeostasis, and that the effect of other variables is to enhance responsiveness, ${ }^{20}$ but it is these other factors which play the major part in precipitating at least the post-menopausal form of the disease. The role of effective loading will be to prepare a skeleton better able to resist these catabolic processes. The anabolic response of some but not all exercise programmes indicates that exercise can be protective, even in these age groups, but there is some uncertainty about the most effective form of exercise. ${ }^{21} 2237$ The predictions arising from the hypothesis may clarify this problem.

\section{Predictions}

Prevention, arrest, and possibly, in its early stages, reversal, of idiopathic osteoporosis will be achieved by a high strain rate exercise regimen which fully uses spinal and appendicular movement ranges. The response will be greater in, but not confined to, young subjects, and the amount of exercise needed will be small - a few minutes exercise a day will suffice. The effect would be enhanced by dynamic extension exercises of the Roman rings type, but other forms of exercise will not necessarily be beneficial to the skeleton.

Static loading, isometric exercise, and habitual low strain rate activities such as walking, will have no preventive effect. Vertical loading cannot provide the axial tension required for trabecular maintenance, and repetitive axial compression, as in jogging, will strengthen cortical bone but could be deleterious to trabeculae.

It is emphasised that these predictions relate to prevention. In a skeleton already osteoporotic, the strain-magnifying effect of increasing movement range could increase the risk of fracture.

I thank Jo Fillery for providing the specimens, Professor Stuart Heap and Drs Fergus Thomson and George Foote for carrying out the computed tomography scans, and am indebted to
George Moltschaniwskyj for calculating the induced strains. I also thank Alex Fraser for the photographs.

1 Cummings SR, Kelsey JL, Nevitt MC, O’Dowd KJ. Epidemiology of osteoporosis and osteoporotic fractures. Epidemiol Rev 1985;7:178-208.

2 Arlot M, Edouard P, Meunier PJ, Neer RM, Reeve J. Impaired osteoblast function in osteoporosis: comparison between calcium balance and dynamic histomorphometry. BMJ 1984;289:517-20.

3 Parfitt AM. Bone remodelling and bone loss: understanding the pathophysiology of osteoporosis. Clin Obstet Gynecol 1987;30:789-811.

4 Kanis JA. Textbook of osteoporosis. London: Blackwell Science, 1996 .

5 Khosla S, Melton LJ, Atkinson EJ, O'Fallon WM, Klee GG, Riggs BL. Relationship of serum sex steroid levels and bone turnover markers with bone mineral density in men and women: a key role for bioavailable estrogen. J Clin Endocrinol Metab 1998;83:2266-74

6 Block JE, Smith R, Glueer C-C, Stieger P, Ettinger B, Genant HK. Models of spinal trabecular bone loss as determined by quantitative computerised tomography. J Bone Miner Res 1989;4:249-57.

7 Arnold JS, Bartley MH, Tont SA, Jenkins DP. Skeletal changes in aging and disease. Clin Orthop 1966;49:17-38.

8 Riggs BL, Melton LJ. Involutional osteoporosis. N Engl J Med 1986;314:1676-86.

9 Meier DE, Orwoll ES, Keenan EJ, Fagerstrom RM. Marked decline in trabecular bone mineral content in healthy men with age: lack of association with sex steroid levels. J Am with age: lack of association
Geriatr Soc 1987;35:189-97.

10 Eriksen EF, Langdahl BL. The pathogenesis of osteoporosis. Horm Res 1997;48(suppl 5):78-82.

11 Villanueva AR, Ilnicki L, Duncan H, Frost HM. Bone and cell dynamics in osteoporosis. Clin Orthop 1966;49:13550

12 Atkinson PJ. Variations in trabecular structure of vertebrae with age. Calcif Tissue Res 1967;1:24-32.

13 Merz WA, Schenk RK. Quantitative structural analysis of human cancellous bone. Acta Anat (Basel) 1970;75:54-66

14 Parfitt AM, Matthews CHE, Villanueva AR, Kleerekoper M, Frame B, Rao DS. Relationships between surface, volume, and thickness of iliac trabecular bone in aging and osteoporosis. J Clin Invest 1983;72:1396-409.

15 Vesterby A, Gundersen HJG, Melsen F, Mosekilde L. Normal post-menopausal women show iliac crest trabecular thickening on vertical sections. Bone 1989;10:333-9.

16 Cann CE, Genant HK. Precise measurement of vertebral mineral content using computerised tomography. J Comput Assist Tomogr 1980;4:493-500.

17 Vernon-Roberts B, Pirie CJ. Healing trabecular microfractures in the bodies of lumbar vertebrae. Ann Rheum Dis 1973;32:406-12.

18 Twomey L, Taylor J, Furniss B. Age changes in the bone density and structure of the lumbar vertebral column. J Anat 1983;136:15-25.

19 Rubin CT, Lanyon LE. Osteoregulatory nature of mechanical stimuli: function as a determinant for adaptive remodelling in bone. J Orthop Res 1987;5:300-310.

20 Lanyon LE. Using functional loading to influence bone mass and architecture: objectives, mechanisms, and relationship with estrogen of mechanically adaptive process in bone. Bone 1996;18:37-44S.

21 Mosley JR. Osteoporosis and bone functional adaptation: mechanobiological regulation of bone architecture in growing and adult bone, a review. J Rehabil Res Dev 2000; 3rowing and

22 Chamay A, Tschantz P. Mechanical influences in bone remodelling. Experimental research on Wolff's law. J Biomech 1972;5:173-80.

23 Ilizarov GA. The tension-stress effect on the genesis and growth of tissues. Clin Orthop 1989;239:263-85.

24 Delloye C, Delefortrie G, Coutelier L, Vincent A. Bone regenerate formation in cortical bone during distraction lengthening. An experimental study. Clin Orthop 1990; 250:34-42.

25 Richards M, Kozloff KM, Goulet JA, Goldstein SA. Increased distraction rates influence precursor tissue composition without affecting bone regeneration. J Bone Miner position without affectir

26 Storey E, Feik SA. Remodelling of bone and bones: effects of altered mechanical stress on caudal vertebrae. J Anat 1985;140:37-48. 
27 Bassett CAL. Biophysical principles affecting bone structure. In: Bourne GH, ed. The biochemistry and physiology of Churches AE, Howlett CR, Waldron KJ. The response of living bone to controlled time-varying load: method and preliminary results. J Biomech 1979;12:35-45.

29 O'Connor JA, Lanyon LE, MacFie H. The influence of strain rate on adaptive bone remodelling. J Biomech 1982, 15:767-81.

30 Torrance AG, Mosley JR, Suswillo RF, Lanyon LE. Noninvasive loading of the rat ulna in vivo induces a strain-related modelling response uncomplicated by trauma or periosteal pressure. Calcif Tissue Int 1994;54: 241-7.

31 Mosley JR, Lanyon LE. Strain rate as a controlling influence on adaptive modelling in response to dynamic loading of the ulna in growing male rats. Bone 1998;23:313-18.

32 Bassey EJ, Ramsdale SJ. Increase in femoral bone density in young women following high impact exercise. Osteoporosis Int 1994;4:72-5.

33 Heinonen A, Kannus P, Sievänen H, Oja P, Pasanen M, Rinne $\mathrm{M}$, et al. Randomised controlled trial of effect of high impact exercise on selected risk factors for osteoporotic

34 Mosley JR, March BM, Lynch J, Lanyon LE. Strain magnitude related changes in whole bone architecture in growing rats. Bone 1997;20:191-8.

35 Rubin CT, Lanyon LE. Regulation of bone formation by applied dynamic loads. J Bone Joint Surg Am 1984;66: 397-402.

36 Bassey EJ. Exercise in primary prevention of osteoporosis in women. Ann Rheum Dis 1995;54:861-2.
37 Lanyon LE, Hampson WGJ, Goodship AE, Shah JS. Bone deformation recorded in vivo from strain gauges attached
to the human tibial shaft. Acta Orthop Scand $1975 ; 46$ : to the he-68.

38 Chandler FA. Stresses in a curved column. J Bone Joint Surg Br 1933;15:214.

39 Rubin CT, Lanyon LE. Limb mechanics as a function of speed and gait. J Exp Biol 1982;101:187-211.

40 Tuttle RH. Apes of the world. Park Ridge: Noyes Publications, 1986:35, 43-6.

41 Hunt KD. Positional behaviour of pan troglodytes in the Mahale mountains and Gombe Stream National Parks, Tanzania. Am J Phys Anthropol 1992;87:83-105.

42 De Vore I. Primate behaviour: field studies of monkeys and apes. New York: Holt, Rinehart and Winston, 1965:384

43 Avis V. Brachiation; the crucial issue for man's ancestry. Southwest J Anthropol 1962;18:119-48.

44 Preuschoft $\mathrm{H}$, Demes B. Biomechanics of brachiation. In : Preuschoft H, Chivers DJ, Brockelman WY, Creel N, eds. The lesser apes: evolutionary and behavioural biology. Edinburgh: Edinburgh University Press, 1984:96-118.

45 Lanyon LE. In vivo bone strain recorded from thoracic vertebrae of sheep. J Biomech 1972;5:277-81.

6 57.

47 White AA, Panjabi MM. The basic kinematics of the human spine. Spine 1978;3:12-19.

48 Murray MP, Drought AB, Kory RC. Walking patterns in normal men. J Bone Joint Surg Am 1964;46:335-60.

49 Alexander CJ. Relationship between the utilisation profile of individual joints and their susceptibility to primary osteoarthritis. Skeletal Radiol 1989;18:199-205 